\title{
Bacteriemia por Comamonas kerstersii en un joven con apendicitis aguda
}

\author{
Comamonas kerstersii bacteremia in a young man with acute appendicitis
}

Rosario Palacio', Laura Cabezas', Cecilia Cornejo y Verónica Seija

'Departamento de Laboratorio de Patología Clínica. Hospital de Clínicas. Facultad de Medicina. Universidad de la República. Montevideo, Uruguay.

No existe conflicto de interés, ni fuente de financiamiento.

Recibido: 10 de agosto de 2019 / Aceptado: 25 de febrero de 2020

\section{Resumen}

Comamonas kerstersii es un bacilo gramnegativo no fermentador, asociado a infecciones intraabdominales. Comunicamos un caso clínico de una bacteriemia por $C$. kerstersii en un joven con una apendicitis aguda. La primera identificación, mediante el sistema Vitek 2 compact (BioMerieux) desde los hemocultivos, fue Comamonas testosteroni. Sin embargo, mediante MALDI-TOF se identificó como C. kerstersii. El paciente fue tratado con ampicilina/sulbactam y luego piperacilina/ tazobactam con una evolución favorable. Dentro del género Comamonas, C. testosteroni ha sido la especie más frecuentemente reportada como causa de infecciones en humanos, describiéndose solo 23 casos de C. kerstersii. Dado los problemas en la identificación fenotípica de estos microorganismos, es posible que algunos de los reportes de $C$. testosteroni pudieran haber correspondido a $C$. kerstersii. Este caso pone en evidencia el rol patógeno de $C$. kerstersii y la importancia de utilizar MALDI-TOF como herramienta diagnóstica en bacilos gramnegativos no fermentadores.

Palabras clave: Comamonas testosteroni; Comamonas kerstersii; bacteriemia.

\section{Introducción}

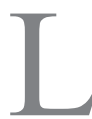

a familia Comamonadaceae incluye los géneros Comamonas, Delftia y Acidovorax ${ }^{1}$. El genero Comamonas spp. fue creado en 1985 e incluía una sola especie, Comamonas terrigena, antes denominada Pseudomonas terrigena. En 1987, Pseudomonas testosteroni y Pseudomonas acidovorans fueron reclasificadas como miembros del género Comamonas ${ }^{1}$. Mas tarde, Comamonas acidovorans fue reclasificada como Delftia acidovorans. Comamonas terrigena incluía tres grupos de aislamientos clínicos, actualmente reconocidos como C. terrigena, Comamonas aquatica y Comamonas

\begin{abstract}
Comamonas kerstersii is a non-fermenting Gram-negative bacillus. It has been associated with intra-abdominal infections. We describe a clinical case of bacteremia caused by $C$. kerstersii in a young man with acute appendicitis. The first identification, using the Vitek2 compact system (BioMerieux) from blood cultures, was Comamonas testosteroni. However, using MALDI-TOF was identified as $C$. kerstersii. The patient was treated first with ampicillin sulbactam and then piperacillin tazobactam with favorable evolution. Within the genus Comamonas, $C$. testosteroni has been the most frequently reported species as a cause of infections in humans, only 23 cases of $C$. kerstersii being described. Given the problems of phenotypic identification of these microorganisms, it is possible that some $C$. testosteroni reports could have corresponded to $C$. kerstersii. This case highlights the pathogenic role of $C$. kerstersii and the importance of using MALDI-TOF as a diagnostic tool for non-fermenting Gramnegative bacilli identification.

Keywords: Comamonas testosteroni; Comamonas kerstersii; bacteremia.
\end{abstract}

kerstersii ${ }^{2}$. A lo largo del tiempo otras especies de origen ambiental se han sumado al género Comamonas ${ }^{3}$. El hábitat de este género comprende el agua, los suelos, la rizósfera y las plantas incluyendo frutas y vegetales. Se trata de bacilos gramnnegativos aerobios, no fermentadores, oxidasa positivos que crecen bien en los medios de cultivo utilizados habitualmente. Raramente causan infección en los seres humanos ${ }^{3}$. Comamonas testosteroni ha sido la especie mas frecuentemente reportada como causa de infecciones en humanos. Su rol patógeno está asociado principalmente a infecciones intraabdominales. Se presenta un caso de bacteriemia causada por $C$. kerstersii en un joven con apendicitis aguda. 


\section{Caso clínico}

Varón de 16 años, sin antecedentes personales a destacar, que consultó en nuestro hospital por dolor abdominal periumbilical que migró hacia la fosa ilíaca derecha, de un día de evolución, acompañado de sensación febril, náuseas y vómitos. Al examen físico se presentó vigil, con temperatura axilar de $40{ }^{\circ} \mathrm{C}$, polipnea de 23 respiraciones por minuto, ritmo cardíaco regular de 160 latidos por minuto y presión arterial de $110 / 60 \mathrm{mmHg}$. Al examen físico, el abdomen era blando, depresible, con dolor a la palpación de hemiabdomen derecho, sin signos de irritación peritoneal. En el hemograma destacó una leucocitosis de 14.020 céls $/ \mathrm{mm}^{3}$, con neutrofilia de 13.180 céls $/ \mathrm{mm}^{3}(94 \%)$, sin otras alteraciones. Se realizó una ecografia abdominal que no identificó el apéndice cecal, y que mostró alteraciones en la ecogenicidad de la grasa, sin líquido libre en la fosa ilíaca derecha. El día de su ingreso se extrajeron dos muestras de hemocultivos y se inició tratamiento antimicrobiano intravenoso con ampicilina/sulbactam a dosis de 1,5 $\mathrm{g}$ asociado a metronidazol $500 \mathrm{mg}$, ambos fraccionados cada seis horas. A las $15 \mathrm{~h}$ del ingreso, con diagnóstico clínico de apendicitis aguda, se realizó una cirugía laparoscópica con exploración de la cavidad abdominal que mostró un apéndice retrocecal ascendente gangrenoso con un absceso apendicular. Se efectuó la liberación del apéndice de la cavidad con apendicectomía retrógrada, evacuación y aspirado del absceso, colocándose un drenaje hacia la cavidad. No se enviaron muestras obtenidas en el acto quirúrgico para estudio microbiológico. Al tercer día del postoperatorio presentó fiebre de $38,5^{\circ} \mathrm{C}$, por lo que se decidió cambiar el tratamiento antibacteriano a piperacilina/tazobactam intravenosa, a dosis de 4,5 g fraccionado cada $6 \mathrm{~h}$, remitiendo la fiebre a partir del cuarto día. Al octavo día se retiró el drenaje. Con buena evolución y habiendo completando 10 días de tratamiento antimicrobiano el paciente fue dado de alta.

En relación al diagnóstico microbiológico, luego de $16 \mathrm{~h}$ de incubación, ambas muestras de hemocultivos emitieron una señal positiva de crecimiento en el sistema BacT/ALERT $^{\circledR}$ (bioMérieux). En la tinción de Gram del caldo de ambas botellas, se observaron bacilos gramnegativos. En ambos hemocultivos se aislaron bacilos gramnegativos que crecieron en agar sangre ovina y agar MacConkey lactosa a $35^{\circ} \mathrm{C}$, con prueba de oxidasa positiva. Se identificó como Comamonas testosteroni, utilizando la tarjeta GN del sistema Vitek 2 compact (BioMerieux). Para confirmar la identificación se envió la cepa al Departamento de Bacteriología y Virología del Instituto de Higiene, Facultad de Medicina. Mediante $\mathrm{Ma}$ trix-Assisted Laser Desorption Ionización-Time of Flight (MALDI-TOF) Bruker se identificó como C. kerstersii, con un score $\geq 2$. Frente a este resultado discordante, se evaluó la capacidad de degradar la pirridonil ß-naftilamida (PYR), cuyo resultado fue negativo y la capacidad de crecimiento a $42{ }^{\circ} \mathrm{C}$ que fue positiva. Ambos resultados fueron congruentes con la identificación de C. kerstersii, tal cual se puede ver en la Tabla $1^{3}$.

El estudio de susceptibilidad antibacteriana se realizó con la tarjeta AST249 del sistema VITEK ${ }^{\circledR} 2$ (bioMérieux) y sus resultados se observan en Tabla 2. Se interpretaron de acuerdo a los puntos de corte de bacilos gramnegativos no fermentadores establecidos por CLSI en $2019^{4}$.

\section{Discusión}

Comamonas testosteroni ha sido la especie más frecuentemente reportada como causa de infecciones en humanos. Se han reportado numerosos aislamientos asociados a infecciones intraabdominales ${ }^{5-10}$. Asimismo, los casos publicados incluyen endocarditis, meningitis y bacteriemia $^{11-16}$. En varios de estos casos no se especifica con cuales pruebas o sistemas comerciales se logró la identificación ${ }^{6,8,13,15}$. En otros, se utilizan diferentes métodos tales como el sistema comercial Vitek 2 compact $^{5,10,12,14}$, el sistema comercial Phoenix ${ }^{7,9}$ y/o galerías API NE $\mathrm{NE}^{7,11,16}$. En nuestro caso se utilizó la tarjeta GN

Tabla 1. Pruebas diferenciales de Comamonas kerstersii/Comamonas testosteroni Comamomas kerstersii Comamonas testosteroni

Crecimiento a $42^{\circ} \mathrm{C}$ $+$

PYR ${ }^{1}$

${ }^{1}$ Capacidad de degradar pirridonil $\beta$-naftilamida.

\begin{tabular}{|lcc|}
\hline \multicolumn{2}{|l|}{ Tabla 2. Susceptibilidad de Comamonas kerstersii } & \\
\hline Antimicrobianos ${ }^{1}$ & CIM $\boldsymbol{\mu} \mathbf{g} / \mathbf{m l}$ & Interpretación \\
Ampicilina/sulbactam & $\leq 2$ & -2 \\
Ceftazidime & 2 & $\mathrm{~S}$ \\
Cefepime & $\leq 1$ & $\mathrm{~S}$ \\
Piperacilina/tazobactam & $\leq 4$ & $\mathrm{~S}$ \\
Meropenem & 0,25 & $\mathrm{~S}$ \\
Imipenem & 0,25 & $\mathrm{~S}$ \\
Gentamicina & 4 & $\mathrm{~S}$ \\
Amikacina & 32 & $\mathrm{I}$ \\
\hline Cotrimoxazol & $\leq 20$ & $\mathrm{~S}$ \\
\hline ISegún CLSI 2019 M100-29th ed. Tabla 2B5. ${ }^{2}$ Sin puntos de corte. & \\
\hline
\end{tabular}


de VITEK ${ }^{\circledR}$ (bioMérieux), que lo identificó como $C$. testosteroni. Dado lo inusual del hallazgo decidimos enviar la cepa a laboratorio de referencia donde, mediante MALDI-TOF, se la identificó como C. kerstersii con un score $\geq 2$. Este valor es considerado válido para la determinación de especie ${ }^{17}$.

Las comunicaciones de infecciones en humanos causadas por $C$. kerstersii alcanzan los 23 casos $^{18-23}$. Esta especie no esta incluída en la base de datos de diferentes sistemas comerciales de identificación fenotípica ${ }^{22}$. Por tanto, es posible que algunos de los reportes existentes de $C$. testosteroni $i^{j-16}$ pudieran haber correspondido a $C$. kerstersii, especialmente los correspondientes a infecciones intraabdominales.

Almuzara y cols ${ }^{18}$ reportaron los primeros cuatro casos de C. kerstersii. Utilizaron diferentes pruebas para llegar a la identificación, incluido VITEK ${ }^{\circledR}$. Con la tarjeta GN obtuvieron resultados de baja discriminación donde una de las posibilidades era $C$. testosteroni salvo en un caso, donde obtuvo una identificación excelente de $C$. testosteroni $^{18}$. En todos los reportes de C. kerstersii ${ }^{18-23}$ la identificación se realizó, al igual que en nuestro caso, mediante MALDI-TOF. En varios casos, se añadió amplificación genética del ARNr $16 \mathrm{~S}$ de manera de confirmar la especie ${ }^{18,20,22}$. Jiang y cols. ${ }^{23}$ reportaron la secuencia completa del genoma de una cepa recuperada de un caso de una peritonitis asociada a diálisis peritoneal.

En cuanto a la susceptibilidad antibacteriana, la cepa aislada se mostró sensible a todos los antibacterianos testados, excepto amikacina, cuya concentración inhibitoria mínima (CIM) fue intermedia $(32 \mathrm{ug} / \mathrm{ml})$. En aislados de C. kerstersii en Argentina ${ }^{18,20}$ se han reportado CIM de 16 $\mathrm{ug} / \mathrm{ml}$ a amikacina. Podrían considerarse CIM elevadas pero están dentro del rango de sensibilidad. En la literatura especializada, se han reportado aislados resistentes a fluoroquinolonas y cotrimoxazol ${ }^{20-22}$. En general, se mantiene susceptibilidad a piperacilina/tazobactam, cefalosporinas de tercera generación y carbapenémicos ${ }^{18,20,21,22}$.

En el caso de ampicilina/sulbactam no existen puntos de corte para la interpretación. Sin embargo, la CIM encontrada $(\leq 2 \mathrm{ug} / \mathrm{mL})$ correspondería a la categoría sensible utilizando los puntos de corte correspondientes a otros grupos de bacilos gramnegativos. Esto se observa en otros casos publicados ${ }^{18,20}$.

Veintidos de los 23 casos donde se aisló $C$. kerstersii correspondieron a infecciones peritoneales, en 16 casos el origen de estas infecciones fue apendicular ${ }^{18-22}$. En la mayoría de los casos se aisló C. kerstersii asociado a otros agentes.

El caso descrito representa el tercer caso de bacteriemia encontrado en la literatura médica. El primero correspondió a un varón de 65 años con una diverticulosis donde se planteó una probable translocación intestinal. El paciente fue tratado con imipenem ${ }^{21}$. El segundo fue un paciente de 31 años, que al igual que el presente caso, correspondió a una bacteriemia secundaria a una perforación apendicular. En este segundo caso, el tratamiento fue cefuroxima más metronidazo ${ }^{22}$. En nuestro caso clínico, el tratamiento fue inicialmente ampicilina/sulbactam y luego cambiado a piperacilina/tazobactam por persistencia de la fiebre.

Si bien la cepa aislada presentó bajos valores de CIM para ambos antimicrobianos, el cuadro clínico de este paciente, en la mayoría de los casos, es de etiología polimicrobiana. Por ello, no podemos descartar la participación de otros agentes infecciosos, resistentes a ampicilina/ sulbactam. Se perdió la oportunidad de encontrar otros potenciales agentes involucrados al no enviar muestra obtenida durante el procedimiento quirúrgico para estudio microbiológico.

Biswas y cols. ${ }^{19}$ comunicaron el aislamiento de C. kerstersii en coprocultivos provenientes de pacientes con diarrea. Esto lleva a la hipótesis de que la exposición ambiental podría llevar a colonización intestinal por $C$. kerstersii ${ }^{19}$. Se plantea entonces que se trataría de un patógeno oportunista.

Tanto el presente caso de bacteriemia por $C$. kerstersii, así como los anteriores publicados ${ }^{21,22}$ demuestran que se le puede encontrar causando infecciones graves. Con la creciente utilización de MALDI-TOF, es probable que se identifiquen más cepas de $C$. kerstersii, lo que nos permita caracterizar mejor los cuadros clínicos en los que participa este agente.

\section{Referencias bibliográficas}

1.- Wen A, Fegan W, Hayward C, Chakraborty S, Sly L. Phylogenetic relationships among members of the Comamonadaceae, and description of Delftia acidovorans (den Dooren de Jong 1926 and Tamaoka et al. 1987) gen. nov., comb. nov. Int J Syst Bacteriol 1999; 49: 567-76.

2.- Wauters G, De Baere T, Willems A, Falsen E, Vaneechoutte M. Description of Comamonas aquatica comb. nov. and Comamonas kerstersii $\mathrm{sp}$. nov. for two subgroups of Comamonas terrigena and emended description of Comamonas terrigena. Int J Syst Evol Microbiol 2003; 53: 859-62.

3.- Procop G W, Church D L, Hall G S, Janda W M, Koneman E W, Schreckenberger P C. Capítulo 7: Bacilos Gram negativos no fermentadores. Koneman Diagnóstico Microbiológico Texto y Atlas. $7^{\circ} \mathrm{ed}$.
Philadelphia: Wolters Kluwer; 2017 , p. 316-431.

4.- Clinical and Laboratory Standards Institute (CLSI). Performance Standards for Antimicrobial Susceptibility Testing. 29th ed. CLSI supplement M100. Wayne, PA: Clinical and Laboratory Standards Institute; 2019.

5.- Farooq S, Farooq R, Nahvi N. Comamonas testosteroni: Is it still a rare human pathogen? Case Rep Gastroenterol 2017; 11: 42-7. doi: 10.1159/000452197. 
6.- Parolin M, Baraldi M, Valentini E, Murer L, Vidal E. Comamonas testosteroni-associated peritonitis in a pediatric peritoneal dialysis patient. World J Nephrol 2016; 5: 220-23. doi: 10.5527/wjn.v5.i2.220.

7.- Khalki H, Deham H, Taghouti A, Yahyaoui G, Mahmoud M. Comamonas testosteroni appendicitis. Med Mal Infect 2016; 46: 168-70. doi: 10.1016/j.medmal.2015.12.009.

8.- Altun E, Kaya B, Taktakoglu O, Karaer R, Paydas S, Balal M, et al. Comamonas testosteroni peritonitis secondary to dislocated intrauterine device and laparoscopic intervention in a continuous ambulatory peritoneal dialysis patient. Perit Dial Int 2013; 33: 576-78. doi: 10.3747/pdi.2013.00007.

9.- $\quad$ Tsui T, Tsao S M, Liu K S, Chen T Y, Wang Y $\mathrm{L}$, Teng Y H, et al. Comamonas testosteroni infection in Taiwan: Reported two cases and literature review. J Microbiol Immunol Infect 2011; 44: 67-71. doi: 10.1016/j. jmii.2011.01.013.

10.- Barbaro D, Mackowiak P, Barth S, Southern P. Pseudomonas testosteroni infections: eighteen recent cases and a review of the literature. Rev Infect Dis 1987; 9: 124-29.

11.- Arda B, Aydemir S, Yamazhan T, Hassan A, Tunger A, Serter D. Comamonas testosteroni meningitis in a patient with recurrent cholesteatoma. APMIS 2003; 111: 474-6.

12.- Cooper G R, Staples E D, Iczkowski K A, Clancy C J. Comamonas (Pseudomonas) testosteroni endocarditis. Cardiovasc Pathol 2005; 14: 145-9.

13.- Le Moal G, Paccalin M, Breux J, Roblot F, Roblot P, Giraudon B. Central venous catheter-related infection due to Comamonas testosteroni in a woman with breast cancer. Scand J Infect Dis 2001; 33: 627-8.

14.- Swain B, Rout S. Comamonas testosteroni bacteraemia in a tertiary care hospital. Indian J Med Microbiol 2015; 33: 602-3. doi: 10.4103/0255-0857.167325.

15.- Orsini J, Tam E, Hauser N, Rajayer S. Polymicrobial bacteremia involving Comamonas testosteroni. Case Rep Med 2014; 2014: 578127. doi: 10.1155/2014/578127.

16.- Farshad S, Norouzi F, Aminshahidi M, Heidari B, Alborzi A. Two cases of bacteremia due to an unusual pathogen, Comamonas testosteroni in Iran and a review literature. J Infect Dev Ctries 2012; 6: 521-5.

17.- Bizzini A, Durussel C, Bille J, Greub G, Prod'hom G. Performance of matrix-assisted laser desorption ionization-time of flight mass spectrometry for identification of bacterial strains routinely isolated in a clinical microbiology laboratory. J Clin Microbiol 2010; 48: 1549-54. doi: 10.1128/JCM.0179409.

18.- Almuzara M N, Cittadini R, Vera Ocampo C, Bakai R, Traglia G, Ramírez M S, et al. Intra-abdominal infections due to Comamonas kerstersii. J Clin Microbiol 2013; 51: 19982000. doi: 10.1128/JCM.00659-13.

19.- Biswas J S, Fitchett J, O’Hara G. Comamonas kertessii and the perforated appendix. J Clin Microbiol 2014; 52: 3134. doi: 10.1128/ JCM.00909-14

20.- Almuzara M, Barberis C, Veiga F, Bakai R, Cittadini R, Vera Ocampo C, et al. Unusual presentations of Comamonas kerstersii infection. New Microbes New Infect 2017; 19: 91-5. doi: 10.1016/j.nmni.2017.07.003.

21.- Opota O, Ney B, Zanetti G, Jaton K, Greub G, Prod'hom G. Bacteremia caused by Comamonas kerstersii in a patient with diverticulosis. J Clin Microbiol. 2014; 52: 1009-12. doi: 10.1128/JCM.02942-13.

22.- Zhou Y H, Ma H X, Dong Z Y, Shen M H. Comamonas kerstersii bacteremia in a patient with acute perforated appendicitis: A rare case report. Medicine (Baltimore) 2018; 97: e9296. doi: 10.1097/MD.0000000000009296. 